\title{
Flexible Dielectric Waveguides with Powder Cores
}

\author{
WILLIAM M. BRUNO, MEMBER, IEEE, AND WILLIAM B. BRIDGES, FELIOW, IEEE
}

\begin{abstract}
Flexible dielectric waveguides have been demonstrated at 10 GHz and 94 GHz using thin-wall polymer tubing filled with low-loss, high-dielectric-constant powders. Absorptive losses of the order of 10 $\mathrm{dB} /$ meter were measured at $94 \mathrm{GHz}$ with nickel-aluminum titanate and barium tetratitanate powder in polytetrafluoroethylene (PTFE) lightweight electrical spaghetti. Bending losses at $94 \mathrm{GHz}$ were negligible for curvature radii greater than $4 \mathrm{~cm}$. Kuhn's theory of three-region cylindrical dielectric waveguide was used to calculate dispersion curves for the lower order modes for several combinations of dimensions and dielectric constants. Good agreement was obtained between experimental and theoretical values of guide wavelength. A scheme is proposed for classifying hybrid modes of three-region guides based on $\left|E_{z} / H_{z}\right|$. For two-region guides, it reduces to Snitzer's familiar scheme.
\end{abstract}

\section{INTRODUCTION}

$\mathrm{M}$ ETAL WAVEGUIDE used at millimeter-wave frequencies is rigid and expensive (typically $\$ 40$ per meter for WR-10 W-band extruded copper waveguide, exclusive of end flanges). An attractive alternative would be an inexpensive, flexible dielectric guide analogous to optical fiber. Flexible millimeter waveguides consisting of cylindrical plastic rods have been demonstrated [1], but these guides were "unclad" and thus subject to loss from contact with nearby objects or supports. A flexible millimeter waveguide with a PTFE core and a foamed PTFE cladding is commercially available from W. L. Gore, Inc. [2], but it is rather bulky and subject to bending losses because the core and cladding have similar dielectric constants. Another clad flexible guide was demonstrated by Shindo and Ohtomo with a polyethylene core, polyfoam cladding, and a polyethylene jacket [3].

An ideal dielectric waveguide would have a small core consisting of a flexible, low-loss material with a large dielectric constant. The cladding would also be flexible and low in loss, and its dielectric constant would be much smaller than that of the core, so that the fields of the guided mode would decrease rapidly with distance in the cladding.

Unfortunately, all flexible solids with low millimeterwave losses, such as PTFE and polypropylene, have small dielectric constants (about 2). If they were used for cladding, we would have no flexible solid for a core; if they were used for the core, we would have no material for the

Manuscript received June 22, 1987; revised December 18, 1987.

W. M. Bruno was with the California Institute of Technology, Pasadena, CA. He is now with TRW, Redondo Beach, CA 90278.

W. B. Bridges is with the California Institute of Technology, Pasadena CA 91125

IEEE Log Number 8819966 cladding. Our solution to this dilemma was to use the polymers as the cladding and a low-loss, high-dielectricconstant solid material in powder form as the flexible core of the waveguide.

In Section II of this paper we discuss the theory of these three-region (core/cladding/surrounding medium) dielectric waveguides and present dispersion curves for parameters typical of our guides. In Section III, we describe the experimental investigation of these powder core guides

\section{THEORY}

\section{A. The Characteristic Equation}

The dielectric tubes employed in this study had wall thicknesses much smaller than a wavelength to allow access to the fields of the guided mode for making guide-wavelength measurements. Consequently, we could not make the simplification common to optical fiber analyses of treating a three-region guide with thick cladding as a two-region guide, ignoring the presence of the outermost region. Furthermore, since the difference in dielectric constant or refractive index between the materials used here for core and cladding was typically large, approximations based on small index differences [4], also common in optical fiber analysis, were generally inapplicable. The exact analytical theory of lossless three-region cylindrical dielectric waveguides, given by Kuhn [5], was used to predict propagation constants for the modes of powderfilled tube guides.

The geometry of a three-region cylindrical dielectric waveguide is shown in Fig. 1. Region 1 is the core of the waveguide, region 2 is the cladding, and region 3 is the space surrounding the waveguide. For the fields of the propagating modes to be well confined to the core of the waveguide, the relative dielectric constants of the three regions must satisfy $\epsilon_{1}>\epsilon_{2}>\epsilon_{3}$. Since the materials are assumed to be lossless in this first-order analysis, $\epsilon_{1}, \epsilon_{2}$, and $\epsilon_{3}$ are real. For a low-loss guide, it is usually sufficient to treat the loss as a perturbation, that is, to ignore its effect on the propagation constant, $\beta(\omega)$.

To find the propagation constants for the modes of three-region cylindrical dielectric waveguide, it is necessary to find the solutions to the wave equation for which the tangential field components are continuous at the two material boundaries. Kuhn's approach was to solve for the forms of the axial fields and then use these in Maxwell's 


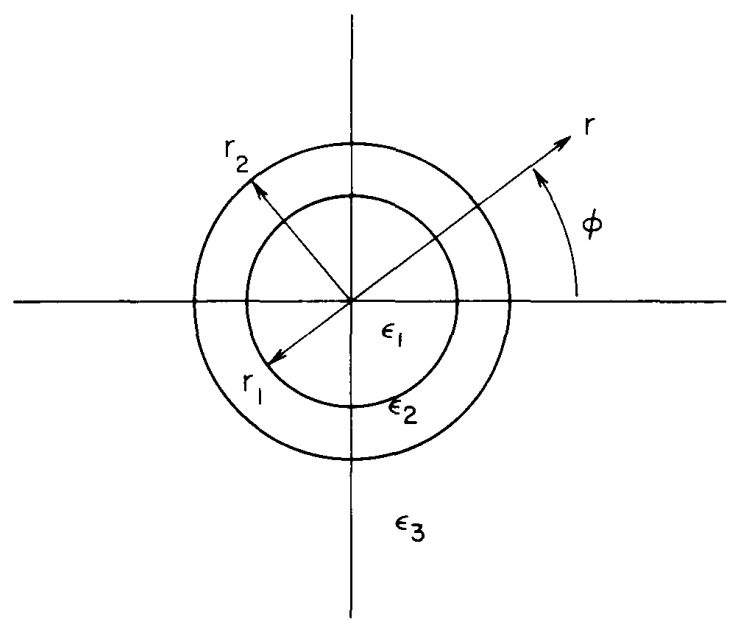

Fig. 1. Cross section of three-region cylindrical dielectric waveguide.

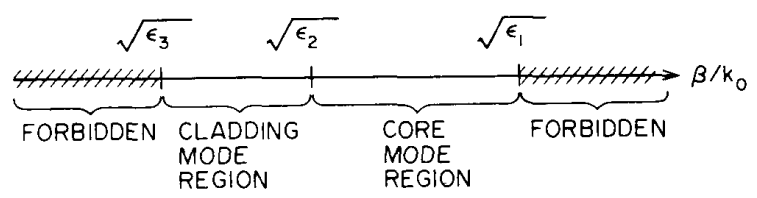

Fig. 2. Core mode and cladding mode regions of a three-region cylindrical dielectric waveguide.

curl equations to derive expressions for the other field components. The characteristic equation giving the propagation constants is then obtained from the equations expressing the continuity of the tangential fields at the boundaries.

The three-region guide solutions require a distinction between mode types that does not arise in the two-region case: Propagating modes are classified as either "core" modes or "cladding" modes [5]. The mathematical functions used to describe the field variations are different for these two classes of modes. If the mode propagation constant $\beta$ satisfies $\beta>k_{0} \sqrt{\epsilon_{2}}$, where $k_{0}=\sqrt{\mu_{0} \epsilon_{0}}$, then the mode is a core mode. Core modes propagate with phase velocities less than $c / \sqrt{\epsilon_{2}}$ but greater than $c / \sqrt{\epsilon_{1}}$. On the other hand, modes for which $\beta<k_{0} \sqrt{\epsilon_{2}}$ are called cladding modes. These modes have phase velocities less than $c / \sqrt{\epsilon_{3}}$ but greater than $c / \sqrt{\epsilon_{2}}$. Physically, cladding modes have larger phase velocities than core modes because a larger fraction of the power of a cladding mode propagates outside the core of the waveguide. Of course, for any propagating mode the propagation constant must lie between the value for a plane wave in free space, $k_{0}$, and a plane wave in the core dielectric, $k_{0} \sqrt{\epsilon_{1}}$. Modes with values of $\beta$ lying outside this interval are cut off (Fig. 2).

Consider the system of cylindrical coordinates shown in Fig. 1, with the $z$ direction coinciding with the longitudinal axis of the waveguide. The form of the $z$ components of the fields of a core mode can be found using separation of variables. Choosing the Bessel functions with the proper behavior at the origin and as $r \rightarrow \infty$, we have

$$
\begin{aligned}
E_{1 z}= & A J_{m}\left(k_{1} r\right) \cos (m \phi) \exp (-j \beta z+j \omega t) \\
H_{1 z}= & B J_{m}\left(k_{1} r\right) \sin (m \phi) \exp (-j \beta z+j \omega t) \\
E_{2 z}= & {\left[C I_{m}\left(k_{2} r\right)+D K_{m}\left(k_{2} r\right)\right] } \\
& \cdot \cos (m \phi) \exp (-j \beta z+j \omega t) \\
H_{2 z}= & {\left[E I_{m}\left(k_{2} r\right)+F K_{m}\left(k_{2} r\right) \sin (m \phi) \exp (-j \beta z+j \omega t)\right.} \\
E_{3 z}= & G K_{m}\left(k_{3} r\right) \cos (m \phi) \exp (-j \beta z+j \omega t) \\
H_{3 z}= & P K_{m}\left(k_{3} r\right) \sin (m \phi) \exp (-j \beta z+j \omega t) .
\end{aligned}
$$

$I_{m}$ and $K_{m}$ are the modified Bessel functions of the first and second kind. Here $m$ is the azimuthal eigenvalue; $A$, $B, C, D, E, F, G$, and $P$ are constants; and the real constants $k_{1}, k_{2}, k_{3}$ are defined by

$$
\begin{aligned}
& k_{1}^{2}=\epsilon_{1} k_{0}^{2}-\beta^{2} \\
& k_{2}^{2}=\beta^{2}-\epsilon_{2} k_{0}^{2} \\
& k_{3}^{2}=\beta^{2}-\epsilon_{3} k_{0}^{2} .
\end{aligned}
$$

The remaining radial and azimuthal field components can be expressed in terms of the $z$ components using Maxwell's curl equations. (See, for example, [6] or [7].) These field components will exhibit similar functional variations with $(r, \phi, z)$ as the $z$ components in each region since they are related to the $z$ components simply by differentiation. Note that for the core modes the $z$ components of the electric and magnetic fields are oscillatory with $r$ in the core region (Bessel function $J_{m}$ ), that they vary smoothly in the cladding region (modified Bessel functions $I_{m}, K_{m}$ ), and that they decay exponentially in the exterior region ( $K_{m}$ only).

By contrast, our definition of a cladding mode requires the constant $k_{2}$ to be imaginary, so we define $k_{2}^{\prime}$ as

$$
\left(k_{2}^{\prime}\right)^{2}=-k_{2}^{2}=\epsilon_{2} k_{0}^{2}-\beta^{2}
$$

while $k_{1}$ and $k_{3}$ remain as before. The field components $E_{2 z}, H_{2 z}$ then become

$$
\begin{aligned}
E_{2 z}= & {\left[C^{\prime} J_{m}\left(k_{2}^{\prime} r\right)+D^{\prime} Y_{m}\left(k_{2}^{\prime} r\right)\right] } \\
& \cdot \cos (m \phi) \exp (-j \beta z+j \omega t) \\
H_{2 z}= & {\left[E^{\prime} J_{m}\left(k_{2}^{\prime} r\right)+F^{\prime} Y_{m}\left(k_{2}^{\prime} r\right)\right] } \\
& \cdot \sin (m \phi) \exp (-j \beta z+j \omega t) .
\end{aligned}
$$

The $z$ components of the fields in regions 1 and 3 retain the same forms as for core modes. Note that the fields in the cladding may become "oscillatory," depending on the cladding thickness.

Equating the tangential field components at the boundaries between regions yields a set of eight linear homogeneous equations for the eight constants $(A, B, C, \cdots, P$.). Setting the secular determinant of this system to zero yields the characteristic equation. This equation is rather complicated and must be solved numerically. Kuhn [5] and Safaai-Jazi and Yip [8] have obtained simplified, but still cumbersome, forms of the characteristic equation by algebraic manipulation of the secular determinant. However, 
these forms cannot be solved analytically either and they do not lend themselves to accurate and unambiguous numerical solution. A simple, fast, and accurate numerical method is to evaluate the secular determinant by Gaussian elimination for trial values of the propagation constant $\beta$.

\section{B. Classification of Modes}

As in the case of a simple two-region dielectric rod, when the azimuthal eigenvalue $m$ is zero, a great simplification is possible. The overall characteristic equation reduces to two much simpler equations. One of these equations corresponds to TM modes and the other corresponds to TE modes [5], [8]. Since $m=0$ the fields of these modes have no circumferential variation. TM and TE modes are designated $\mathrm{TM}_{01}, \mathrm{TM}_{02}, \mathrm{TM}_{03}, \cdots$ and $\mathrm{TE}_{01}, \mathrm{TE}_{02}, \cdots$, where the second subscript gives the order in which the modes cut on (i.e., the propagation constant $\beta$ becomes real) to become guided as the frequency is increased.

Again, as in the case of the two-region dielectric rod, all modes for which $m$ is not zero are termed "hybrid" modes because longitudinal components of $E$ and $H$ are simultaneously present. Several methods have been proposed [5], [8] for classifying hybrid modes for three-region guide, but no method has been universally accepted. Most schemes classify hybrid modes into one of two categories, $\mathrm{HE}_{m n}$ and $\mathrm{EH}_{m n}$. However, the criterion used to determine whether a mode is $\mathrm{HE}$ or $\mathrm{EH}$ is still a subject of controversy.

Most schemes for classifying hybrid modes of threeregion guide are based on schemes previously applied to simple dielectric rods, which we review briefly here. One of the earliest schemes for the dielectric rod was proposed by R. E. Beam in 1949 [9]. Beam's method is based on the relative contributions of $E_{z}$ and $H_{z}$ to a transverse field component at some reference point. If $E_{z}$ makes the larger contribution, the mode is designated $\mathrm{EH}$. If the contribution from $H_{z}$ is larger, the mode is classified as HE. In this scheme, the choice of the names $\mathrm{HE}$ and $\mathrm{EH}$ is reasonable because they are meant to express the hybrid character of the modes and to indicate which longitudinal field component is "more significant" in some sense. In addition, there is historical precedent for this choice of names. They are adapted from names given to modes of metal guides: TE modes of metal guides have been called $\mathrm{H}$ modes and TM modes have been called E modes, particularly in the British literature.

Although the spirit of this naming scheme makes sense, Beam's method is still arbitrary because it depends on the particular transverse component chosen to judge the relative importance of $H_{z}$ and $E_{z}$. Objecting to the arbitrariness of Beam's method, Snitzer [10] proposed a scheme for dielectric rods based on a factorization of the characteristic equation into the form $F 1(\beta) * F 2(\beta)=0$. Since all modes result from either $F 1=0$ or $F 2=0$, the factorization divides the modes into two sets. Snitzer observed two differences between the sets. First, modes stemming from $F 1=0$ obey different cutoff conditions than ones associ- ated with $F 2=0$. Second, the sign of the amplitude coefficient ratio $A / B$ (c.f. (1)) is negative for hybrid modes belonging to one set and positive for hybrid modes from the other set. Since it had become conventional by this time to refer to the fundamental mode as $\mathrm{HE}_{11}$, Snitzer proposed that all hybrid modes for which the sign of $A / B$ is the same as for the fundamental be designated $\mathrm{HE}_{m n}$. Hybrid modes for which $A / B$ has the opposite sign were to be designated $\mathrm{EH}_{m n}$. Here $n$ is the order in which a mode of given class (either $\mathrm{HE}$ or $\mathrm{EH}$ ) and azimuthal eigenvalue cuts on to become guided as frequency is increased.

Although Snitzer's scheme allows the unambiguous classification of hybrid modes into two sets, the use of the names $\mathrm{HE}$ and $\mathrm{EH}$ is at variance with Beam's scheme: modes called $\mathrm{HE}$ do not have longitudinal $H$ dominant over longitudinal E. Similarly, EH modes are not "E-like." In fact, the opposite is true [7], [11]. The magnitude of $A / B$ in normalized units of impedance is always greater than unity for hybrid modes of the dielectric rod designated HE by Snitzer and less than unity for modes called EH. Hence, Snitzer's HE modes are truly "E-like" and the EH modes are "H-like."

Other classification schemes have been proposed for the dielectric rod [12], [13]. The most commonly used of these other methods [13], [14] is based on cutoff conditions of hybrid modes. It results in a classification of hybrid modes identical to Snitzer's.

In the case of the dielectric rod, Snitzer's method and its equivalents have gained wide acceptance [7], [11], [14]. For three-region guide, however, no classification scheme has yet received wide acceptance. Following Snitzer, Kuhn [5] used the sign of $A / B$ to classify hybrid modes of threeregion guide as either $\mathrm{HE}_{m n}$ or $\mathrm{EH}_{m n}$. However, he does not show the existence of any important physical differences between hybrid modes for which the sign of $A / B$ is different. Since the sign of $A / B$, in itself, is not particularly significant, the separation of hybrid modes into two categories using this criterion is not meaningful.

Safaai-Jazi and Yip [8] have proposed a classification scheme based on a factorization of the characteristic equation of three-region guide into a form $F 3(\beta) * F 4(\beta)=0$. Hybrid modes whose propagation constants are found from $F 3(\beta)=0$ are designated $\mathrm{HE}_{m n}$ and those whose propagation constants are given by $F 4(\beta)=0$ are called $\mathrm{EH}_{m n}$. Here again, the authors do not show any general physical differences between modes in each of the two classes. Furthermore, there is more than one way to express the characteristic equation of three-region guide as a product of two functions of $\beta$ set to zero. Different factorizations may result in different classifications of the modes.

We propose here still another scheme for classifying hybrid modes of three-region dielectric waveguide, one based on physically meaningful differences, namely, that the magnitude of the ratio of the longitudinal fields be compared to the wave impedance of a plane wave traveling at the same phase velocity. We define a wave imped- 
ance $Z$ :

$$
Z=\frac{\sqrt{\frac{\mu_{0}}{\epsilon_{0}}}}{\left(\beta / k_{0}\right)}=\frac{377}{\left(\beta / k_{0}\right)} \quad \text { Ohms. }
$$

The ratio $|A / B|$ is, in some sense, the magnitude of the ratio of $E_{1 z}$ to $H_{1 z}$, the longitudinal fields in the core region. If $|A / B|$ evaluated far above the cutoff frequency is greater than $Z$, then the mode is to be called $\mathrm{HE}_{m n}$. Modes for which $|A / B|$ is less than $Z$ will be $\mathrm{EH}_{m n}$. When evaluated far above cutoff, $|A / B| / Z$ approaches unity for all hybrid modes. The classification scheme proposed here is based on whether unity is approached from above or below.

According to this scheme, hybrid modes can be classified unambiguously into two physically distinct categories whose names reflect the distinction. The fundamental mode is still the $\mathrm{HE}_{11}$ mode. Although it would be preferable for historical reasons to have the first letter in the name of a hybrid mode, rather than the second, indicate the "dominant" longitudinal component, such a choice would change the fundamental mode to " $\mathrm{EH}_{11}$." As it stands, our proposed scheme reduces to Snitzer's well-accepted scheme for the simple dielectric rod.

\section{Numerical Results}

Computer programs were written which solved the three-region characteristic equation for $m=1\left(\mathrm{HE}_{1 n}\right.$ and $\mathrm{EH}_{1 n}$ modes) and $m=0$ ( $\mathrm{TE}_{0 n}$ and $\mathrm{TM}_{0 n}$ modes) by using Gaussian elimination to evaluate the secular determinant for trial values of propagation constant. Examples of computed values for propagation constant for different parameters appropriate to our $10 \mathrm{GHz}$ and $94 \mathrm{GHz}$ waveguides are given in Section III. As reported there, the agreement between theoretical and experimental values was excellent.

Here we present theoretical dispersion curves otained with the programs for three-region dielectric waveguides useful at microwave frequencies. These guides have much thinner cladding regions $\left(r_{2}=1.25 r_{1}\right)$ than typical optical fibers. In addition, the dielectric constant difference between core and cladding, $\epsilon_{1}-\epsilon_{2}$, is much larger than that in typical optical fibers.

Sets of dispersion curves are given for $\epsilon_{1}$ of 4,8 , and 12 (Figs. 3 through 5). For each set, $\epsilon_{3}$ is 1 (air) and $\epsilon_{2}$ is 2.08 (PTFE). The abscissa is $k_{0} r_{1}=2 \pi f r_{1} / c$. It may be thought of either as frequency for a guide with fixed core radius, $r_{1}$, or as core radius for operation at a fixed frequency, $f$.

The most noticeable difference between Figs. 3 through 5 and typical dispersion curves of two-region guides is the order of the mode cutoff frequencies. For two-region guides, the $\mathrm{TE}_{0 n}$ and $\mathrm{TM}_{0 n}$ modes have identical cutoffs, as do the $\mathrm{EH}_{1, n}$ and $\mathrm{HE}_{1, n+1}$ modes. However, for threeregion guide, these pairs of modes do not have identical cutoffs, as shown in Figs. 3 through 5. Values of $k_{0} r_{1}$ at cutoff are given in Table I.

Another difference between Figs. 3 through 5 and typical dispersion curves of two-region guides has to do with

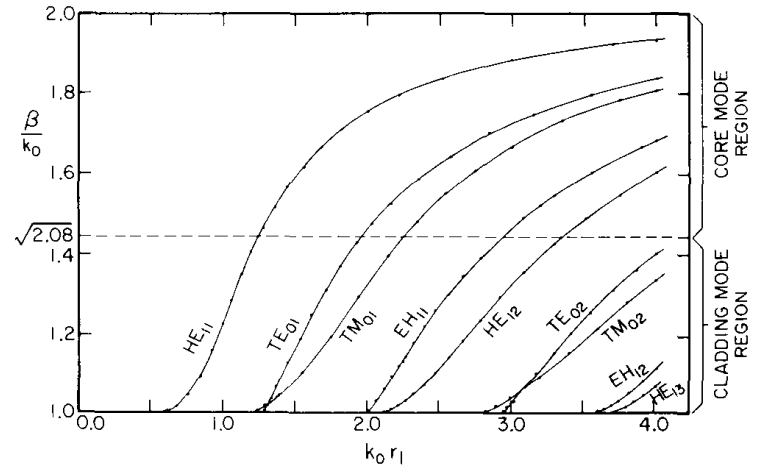

Fig. 3. Dispersion curves of three-region cylindrical dielectric waveguide. $\epsilon_{1}=4, \epsilon_{2}=2.08, \epsilon_{3}=1 . r_{2}=1.25 r_{1}$.

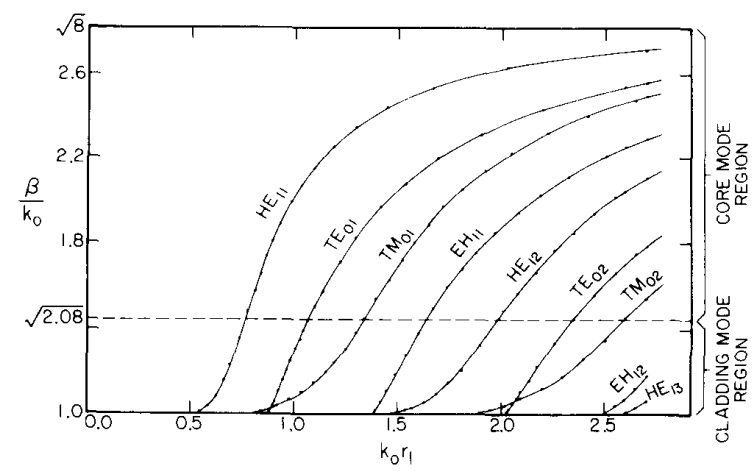

Fig. 4. Dispersion curves of three-region cylindrical dielectric waveguide. $\epsilon_{1}=8, \epsilon_{2}=2.08, \epsilon_{3}=1 . r_{2}=1.25 r_{1}$.

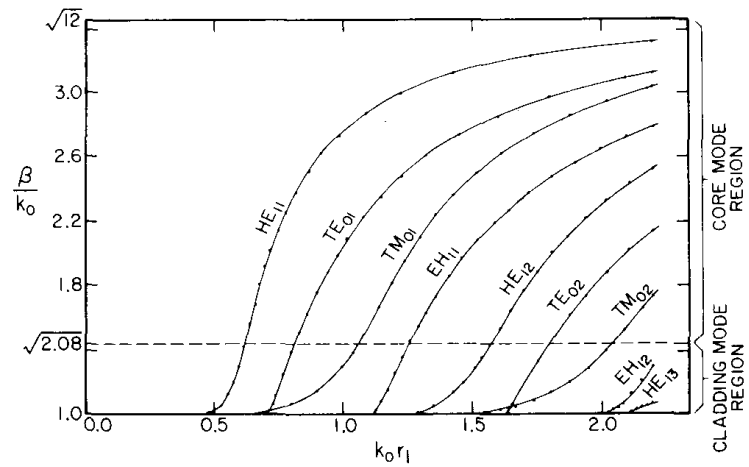

Fig. 5. Dispersion curves of three-region cylindrical dielectric waveguide. $\epsilon_{1}=12, \epsilon_{2}=2.08, \epsilon_{3}=1 . r_{2}=1.25 r_{1}$.

the crossovers of the $\mathrm{TE}_{0 n}$ and $\mathrm{TM}_{0 n}$ modes in Figs. 3 through 5. (The $\mathrm{TE}_{0 n}$ and $\mathrm{TM}_{0 n}$ modes of dielectric rods do not cross.) Safaai-Jazi and Yip [15] have reported that these modes also cross for three-region optical guides where the refractive index difference between core and cladding is small. (Their results were obtained from the exact three-region analysis, not an approximation based on small refractive index difference.) Hence, these crossovers must be attributable to finite cladding thickness. 


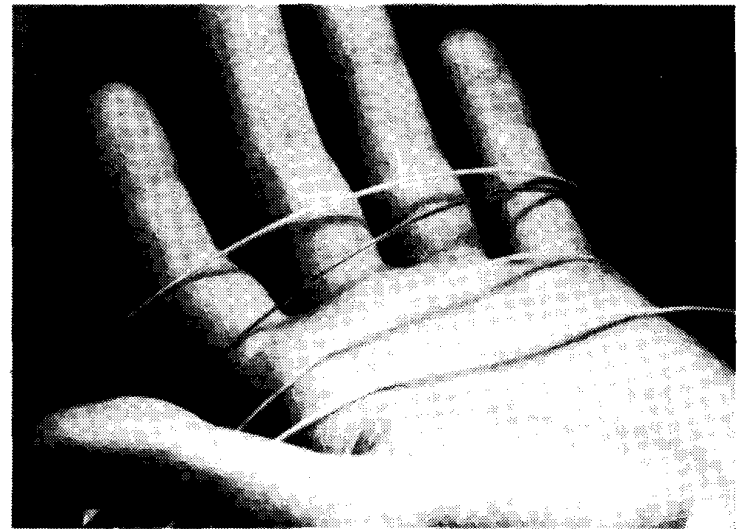

Fig. 6. Samples of $W$-band dielectric waveguide made by filling PTFE spaghetti with various low-loss dielectric powders.

\section{EXPERIMENTAL WORK ON THREE-REgION GUIDES}

Flexible dielectric waveguides were demonstrated at both $10 \mathrm{GHz}$ and $94 \mathrm{Ghz}$ by filling hollow, low-dielectric-constant polymer tubes with low-loss, high-dielectric-constant powders. Flexible guides with losses as low as $0.12 \mathrm{~dB} / \mathrm{cm}$ were demonstrated at $94 \mathrm{GHz}$. These guides also exhibited neglible bending loss for radii of curvature greater than 4 $\mathrm{cm}$. Fig. 6 is a photograph of some samples of $W$-band flexible guide made by this technique.

\section{A. $10 \mathrm{GHz}$ Modeling Experiments}

Our first dielectric waveguides made by filling flexible hollow tubes with dielectric powders were designed for 10 $\mathrm{GHz}$ to avoid inaccuracies due to the small guide dimensions at $94 \mathrm{GHz}$. In addition, the dielectric properties of the powders were known at $10 \mathrm{GHz}$, so the guides could be compared with theory.

The powders used for the high-dielectric-constant core were Emerson and Cumming Ecco-flo powder, Trans-Tech D-30 nickel-aluminum titanate, and Trans-Tech D-38 barium tetratitanate. The particles of the D-30 and D-38 powders ranged in size from $43 \mu \mathrm{m}$ to $100 \mu \mathrm{m}$. Trans-Tech's literature gives $\epsilon=31$ and $\tan \delta<0.0002$ for solid D-30 at $10 \mathrm{GHz}$, and $\epsilon=37$ and $\tan \delta<0.0005$ for solid D-38 at 6 $\mathrm{GHz}$. They do not specify the dielectric properties of the powders. The Ecco-flo powder is specified by Emerson and Cumming to have $\epsilon=12$ and $\tan \delta=0.0007$ at 10 $\mathrm{GHz}$.

In order to design a dielectric waveguide with a powder core, it is necessary to know how the dielectric constant of the powder will vary with the packing density. This relationship was determined for each powder at $10 \mathrm{GHz}$ by using the shorted waveguide technique [16] to the measure dielectric constant. A typical plot of dielectric constant versus density (nickel-aluminum titanate) is given in Fig. 7. Over the density range shown, the dielectric constant is virtually linear with density (although the curve does not pass through the point $(0,1)$ ).
TABLE I

Calculated Values of $k_{0} r_{1}$ at Cutoff for Three-Region GUIDE WITH $r_{2}=1.25 r_{1}$ AND $\epsilon_{3}=1$

\begin{tabular}{|c|c|c|c|}
\hline Mode & $\varepsilon_{1} / \varepsilon_{2}=4 / 2.08$ & $\varepsilon_{1} / \varepsilon_{2}=8 / 2.08$ & $\varepsilon_{1} / \varepsilon_{2}=12 / 2.08$ \\
\hline$T H_{n}$, & 1.24 & 0.82 & 0.65 \\
\hline$T F_{n}$, & 1.28 & 0.88 & 0.71 \\
\hline $\mathrm{EH}_{1}$ & 2.02 & 1.34 & 1.09 \\
\hline$H E_{1:}$ & 2.07 & 1.42 & 1.15 \\
\hline TMn ? & 2.77 & 1.86 & 1.49 \\
\hline$T F_{n ?}$ & 2.92 & 2.02 & 1.63 \\
\hline EH, & 3.53 & 2.39 & 1.93 \\
\hline$H E_{1}$ ? & 3.70 & 2.56 & 2.08 \\
\hline
\end{tabular}

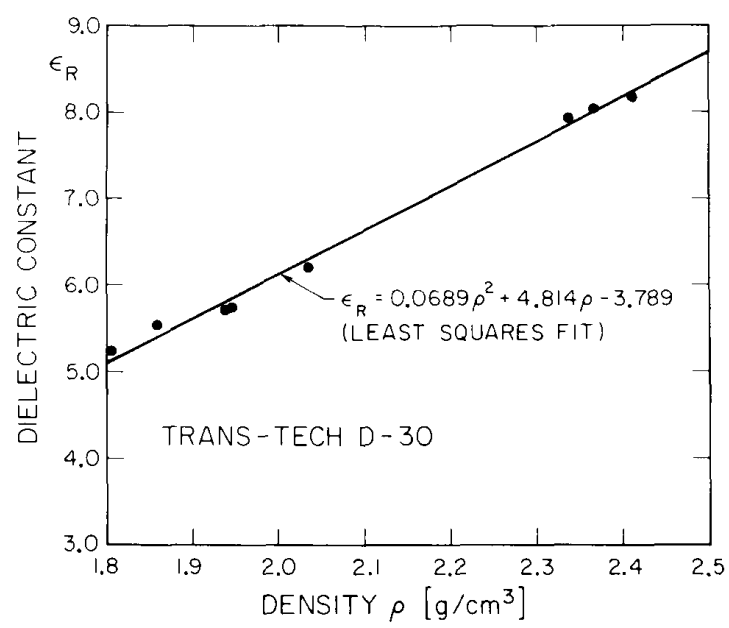

Fig. 7. Relative dielectric constant versus density for Trans-Tech D-30 powder. 70 percent of particles between $100 \mu \mathrm{m}$ and $43 \mu \mathrm{m}, 30$ percent less than $43 \mu \mathrm{m}$.

TABLE II

Dielectric Properties of Tubing Materials at $10 \mathrm{GHz}$

\begin{tabular}{lcc}
\hline Material & $\varepsilon$ & $\tan \delta$ \\
\hline & & \\
PTFE & 2.08 & .0004 \\
polyethylene & 2.25 & .0004 \\
Corning 7740 Pyrex & 4.52 & .0085
\end{tabular}

Data from A. R. Von Hippel, Dielectric Materials and Applications New York: Wiley, 1958, pp. 301-370.

The cladding materials used were PTFE, polyethylene, and Corning 7740 glass (Pyrex). The dielectric properties of these materials at $10 \mathrm{GHz}$ as given by von Hippel [17] are shown in Table II. Although the Pyrex tubes were inflexible, they were useful for making accurate guide wavelength and attenuation measurements.

Each waveguide was made by filling a tube with powder and plugging the ends with polyfoam. The inner diameter 
TABLE III

COMPARISON OF MEASURED AND THEORETICAL GUIDE

WAVElengthS for $\mathrm{HE}_{11}$ MODE OF Three-Region Guide AT $X$-BAND

\begin{tabular}{|c|c|c|c|c|c|c|c|c|c|}
\hline \multirow{2}{*}{$\begin{array}{l}\text { Core } \\
\text { Radius } \\
(\mathrm{cm})\end{array}$} & \multirow{2}{*}{$\begin{array}{l}\text { Cladding } \\
\text { Radius } \\
(\mathrm{cm})\end{array}$} & \multirow{2}{*}{$\begin{array}{l}\text { Freq. } \\
\text { (GHz) }\end{array}$} & \multirow{2}{*}{$\begin{array}{c}\lambda_{0} \\
(\mathrm{~cm})\end{array}$} & \multicolumn{2}{|c|}{ Core } & \multicolumn{2}{|c|}{ Cladding } & \multicolumn{2}{|c|}{ Guide Wavelength } \\
\hline & & & & Mat'l. & $\varepsilon$ & Mat' 1 & $\varepsilon$ & $\begin{array}{l}\text { Meas } \\
(\mathrm{cm})\end{array}$ & $\begin{array}{l}\text { Theo } \\
\text { (cm) }\end{array}$ \\
\hline 0.33 & 0.45 & 10.000 & 3.00 & 1 & 7.62 & A & 4.52 & 2.05 & 2.06 \\
\hline 0.25 & 0.35 & 10.000 & 3.00 & 2 & 13.45 & A & 4.52 & 2.30 & 2.19 \\
\hline 0.26 & 0.30 & 10.000 & 3.00 & 2 & 11.40 & B & 2.08 & 2.88 & 2.86 \\
\hline 0.26 & 0.30 & 11.311 & 2.65 & 2 & 11.40 & B & 2.08 & 2.14 & 2.09 \\
\hline 0.30 & 0.40 & 10.940 & 2.74 & 2 & 13.02 & C & 2.25 & 1.38 & 1.32 \\
\hline 0.32 & 0.47 & 9.794 & 3.06 & 2 & 12.39 & c & 2.25 & 1.71 & 1.65 \\
\hline
\end{tabular}

Material 1 is nickel-aluminum titanate (Trans-Tech D-30).

Material 2 is Emerson and Cuming Ecco-flo powder.

Material A is Corning 7740 Pyrex glass.

Material B is PTFE.

Material $\mathrm{C}$ is polyethylene.

of the tube was chosen so that the $\mathrm{HE}_{11}$ mode would propagate with a wavelength significantly smaller than the free-space wavelength. Coupling was achieved by inserting one end of the tube into a flared section of WR-90 rectangular metal waveguide (a commercial $\mathrm{TE}_{10}$ rectangular to $\mathrm{TE}_{11}$ circular metallic waveguide transition). This metal waveguide transition was used because the transverse fields of the $\mathrm{TE}_{11}$ circular mode of metal waveguide [18] are known to be similar to those of the $\mathrm{HE}_{11}$ mode of a cylindrical dielectric rod. (Although surprisingly good coupling could also be obtained by inserting the dielectric guide directly into the open-ended WR-90.) The dielectric waveguide was supported inside the flared section with a form-fitting polyfoam insert. With the waveguide inserted to the proper depth (determined by trial and error), the reflected power was less than 10 percent, sufficiently small to measure guide wavelength accurately. In addition, there was no detectable radiation field away from the coupler and waveguide. A metal perturber placed a few $\mathrm{mm}$ away from the dielectric guide, outside the volume of the $\left(\mathrm{HE}_{11}\right)$ guided mode, caused no change in reflected power. Finally, lossy foam was wrapped around the tube at the far end to prevent reflections.

Guide wavelength measurements were made by sliding a metal washer along the full length of the guide and observing the periodic variation in reflected power, as indicated schematically in Fig. 8 . The small reflection from the perturber changes phase as the perturber moves, and interferes with the small reflection from the coupler. The measured guide wavelengths were significantly less than that for free-space propagation, indicating that the $\mathrm{HE}_{11}$ mode was well confined. Table III shows that the measured wavelengths were in excellent agreement with those predicted by the theory of lossless three-region cylindrical dielectric waveguide, as presented in Section II. The values of $\epsilon_{\text {core }}$ listed in Table III were determined by using the $\epsilon$

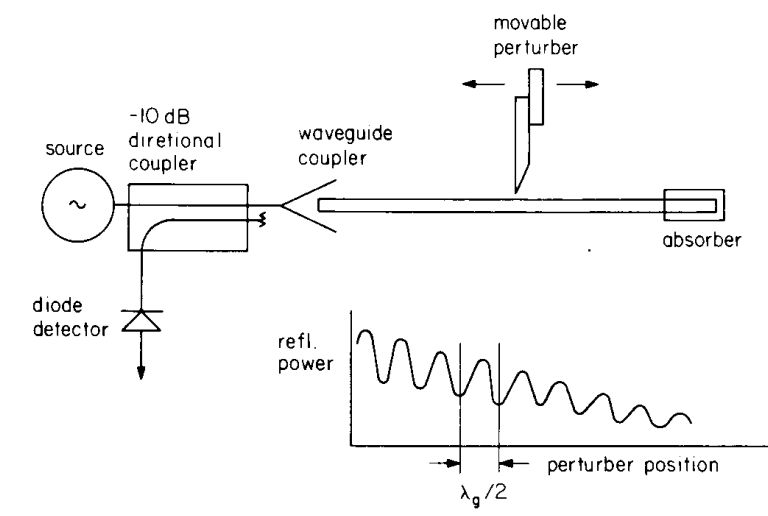

Fig. 8. Schematic of setup for guide wavelength measurement. Also shown is a curve of reflected power versus perturber position.

versus density data and determining the density of the powder in the tube by precision weight measurement.

Preliminary transmission and bending loss measurements were made on powder-filled tubes at $10 \mathrm{GHz}$ by measuring directly the power transmitted to the far end of the guides. The transmitted power was determined by inserting the far end into a section of rectangular metal waveguide connected to a diode detector. These measurements indicated that the transmission and bending losses were low. For example, the total insertion loss, including any coupling losses, for a straight polyethylene tube with length equal to $1 \mathrm{~m}$ and inner diameter equal to $0.6 \mathrm{~cm}$ was $4 \mathrm{~dB}$ when the tube was filled with Ecco-flo powder having dielectric constant equal to $12.8 \pm 0.1$. Bending this guide into circular arcs caused less than a $0.2 \mathrm{~dB}$ increase in loss for curvature radii greater than $28 \mathrm{~cm} \pm 1 \mathrm{~cm}$. Since we were primarily interested in millimeter-wave guides, we did not pursue this investigation far enough at $10 \mathrm{GHz}$ to determine precisely the magnitude of these losses. Instead 
we began a program to build and test flexible $94 \mathrm{GHz}$ powder-filled tube waveguides.

\section{B. $94 \mathrm{GHz}$ Experiments}

Flexible dielectric waveguides for operation at $94 \mathrm{GHz}$ were made by filling PTFE tubes (18-23 AWG lightweight ${ }^{1}$ electrical spaghetti) with dielectric powders. The guides were "designed" using the theory of lossless three-region cylindrical dielectric waveguide so that the $\mathrm{HE}_{11}$ mode would be significantly slowed. In order to use the theory, the dielectric constants of the powders were needed. We used the values measured at $10 \mathrm{GHz}$ because of the difficulty of controlling the length of a powder sample sufficiently accurately to measure its dielectric constant at $94 \mathrm{GHz}$ with the shortest-waveguide technique. The dielectric constant of a powder composed of low-loss dielectric material should not vary much between $10 \mathrm{GHz}$ and 94 $\mathrm{GHz}$ if the powder grains are small relative to the wavelength in the material at $94 \mathrm{GHz}$ [19].

Coupling to metal waveguide was achieved by inserting the end of the dielectric tube into a slightly flared section of WR-10 metal waveguide. As before, guide wavelength measurements were made by sliding a metal perturber along the length of the waveguide and observing the periodic variation in reflected power (Fig. 8).

The Ecco-flo powder proved to be so lossy at $94 \mathrm{GHz}$ that only surface waves would propagate along PTFE tubes filled with this powder. These waves were the same kind of $v \approx c$ waves that were previously observed on KRS-5 guides [20].

We initially tried the same D-30 and D-38 powders used at $X$-band, but were unable to obtain uniform wavelengths along the guides. The measured wavelengths varied by as much as 100 percent with distance along the guide. We believe that one of the factors contributing to this wavelength variation was the random variation with length of the cross-sectional dimensions and circularity of the small (21,22, 23 AWG), commercial grade spaghetti supporting these powders. Another source of error was that the small size of the tubes made them difficult to fill uniformly with powder. Finally, the powder-filled tubes were so flexible that they would bend during the measurement, making the length of a period difficult to measure accurately.

The foregoing difficulties were overcome by using powders with smaller grain size. These powders were Trans-Tech MCT-40 magnesium-calcium titanate, D-30 nickel-aluminum titanate, and D-8512, an "improved" barium tetratitanate. ${ }^{2}$ For each of these powders, all particles were less than $43 \mu \mathrm{m}$ in size. Since these powders had smaller grain sizes and thus smaller packing fractions, they had lower dielectric constants than the others, allow-

${ }^{1}$ PTFE electrical spaghetti is available in three types, according to wall thickness. "Standard wall" spaghetti has the thickest walls, followed in decreasing order of thickness by "thin wall" and "lightweight."

${ }^{2}$ Trans-Tech states that D-8512 has lower loss than D-38 and also a smaller thermal coefficient of dielectric constant. Otherwise, we do not know the nature of the "improvement."
TABLE IV

Comparison of Measured and Theoretical Guide WAVELENGTHS FOR HE HI $_{11}$ MODE OF THREE-REgION GUIDE AT $W$-BAND

\begin{tabular}{lccccc}
\hline Powder $\begin{array}{c}\text { Core } \\
\text { Radulus } \\
\text { (mm) }\end{array}$ & $\begin{array}{c}\text { Freq. } \\
\text { (GHz) }\end{array}$ & Ecnc & \multicolumn{2}{c}{$\begin{array}{c}\text { Guide Vavelength } \\
\text { Meas. } \\
\text { (mm) }\end{array}$} & $\begin{array}{c}\text { Theo. } \\
\text { (mm) }\end{array}$ \\
\hline D-30 & .43 & 94.78 & 5.60 & 2.48 & 2.42 \\
D-8512 & .43 & 94.72 & 5.45 & 2.5. & 2.47 \\
D-8512 & .48 & 94.75 & 5.83 & 2.21 & 2.10 \\
D-8512 & .53 & 94.10 & 4.79 & 2.06 & 2.24 \\
\hline
\end{tabular}

Cladding material is PTFE, $\epsilon=2.08$.

Cladding thickness is $0.15 \mathrm{~mm}$.

The free-space wavelength is approximately $3.2 \mathrm{~mm}$.

ing us to use larger diameter spaghetti $(18,19,20 \mathrm{AWG})$ while still allowing propagation of only the fundamental $\mathrm{HE}_{11}$ mode. Thus, the problems associated with small tubing, dimensional imperfections, packing irregularities, and excessive flexibility were all reduced significantly. The smaller particles also made it easier to pack these powders uniformly in the tubes. As a result of these improvements, the measured guide wavelengths agreed well with the theoretical values for the $\mathrm{HE}_{11}$ mode, as shown in Table IV. For the guides represented in Table IV, there were no beats in the pattern of reflected power versus perturber position, indicating that the guides were single-mode, as intended.

Total transmission attenuation measurements were made by comparing the power received with a diode detector at the far end of the waveguide with the power incident on the guide. Power was coupled from the far end of the dielectric waveguide by inserting it into a flared section of metal waveguide connected to the detector. The incident power was determined by removing the dielectric waveguide and the flared coupling sections and connecting the detector directly to the WR-10 metal waveguide. A second Schottky diode detector connected to a small $W$-band horn antenna was used as a movable probe to determine that there was an insignificant amount of radiation from the couplers and waveguide. Also, the power reflected back into the metal waveguide from the feed coupler was approximately $-20 \mathrm{~dB}$ below the incident power. Thus, we concluded that there was very little power lost in coupling by reflection or radiation, so that the ratio of the incident power to the power detected at the far end represents a reasonable measure of the true dielectric waveguide loss. The loss per unit length is then this loss divided by the length of the dielectric waveguide, typically $30 \mathrm{~cm}$. Table V gives the results of attenuation measurements on a few straight powder-filled Teflon tubes.

Initial bending loss measurements were made using the same setup as for attenuation measurements on straight guides (Fig. 9). The plane of bending was perpendicular to the (vertical) plane of polarization of the $\mathrm{HE}_{11}$ mode. A problem encountered during these measurements was that the ends of the Teflon tubes tended to change position 
TABLE $V$

AtTenuation of Straight MM-WaVe GUides

\begin{tabular}{lccccc}
\hline Powder & $\begin{array}{c}\text { Core } \\
\text { Radius } \\
\text { (mm) }\end{array}$ & $\begin{array}{c}\text { Freq. } \\
\text { (GHz) }\end{array}$ & Ernr. & $\begin{array}{c}\text { Measured } \\
\text { Guide } \\
\text { Wavelength } \\
\text { (mm) }\end{array}$ & $\begin{array}{c}\text { Loss } \\
\text { (dB/Cm) }\end{array}$ \\
\hline D-30 & .43 & 94.78 & 5.60 & 2.48 & .12 \\
D-8512 & .43 & 94.72 & 5.45 & 2.55 & .13 \\
D-8512 & .48 & 94.75 & 5.83 & 2.21 & .14 \\
MCT-40 & .53 & 94.08 & 4.48 & 2.12 & .26 \\
\hline
\end{tabular}

Cladding material is PTFE, $\epsilon=2.08$.

Cladding thickness is $0.15 \mathrm{~mm}$.

The theoretical loss of silver or copper WR-10 waveguide is approximately $0.025 \mathrm{~dB} / \mathrm{cm}$ at $94 \mathrm{GHz}$; however, loss in typical pieces may be $0.05 \mathrm{~dB} / \mathrm{cm}$ or more.

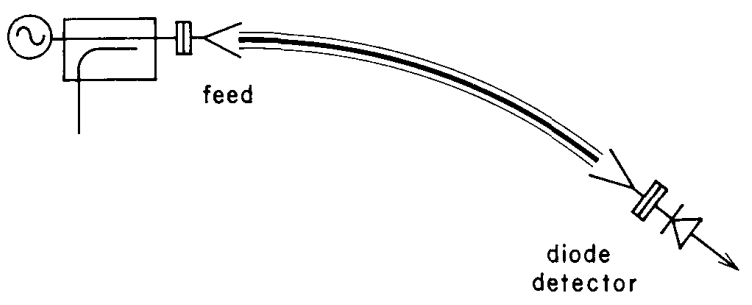

Fig. 9. Schematic representation of the setup for making attenuation and bending loss measurements.

inside the flared metal waveguide couplers when the tubes were bent in arcs with radius less than about $4 \mathrm{~cm}$. This movement changed the quality of the coupling between the dielectric waveguide and the metal waveguides, making it difficult to obtain accurate measurements of bending losses. We did not attempt to refine the mechanical structure to improve these measurements since we had already begun similar measurements on precision-machined rigid rectangular dielectric guides [19], [24]. However, when the tubes were bent into circles with curvature radius greater than about $4 \mathrm{~cm}$, bending losses were immeasurably small, given the $0.2 \mathrm{~dB}$ measurement uncertainty.

A straightforward comparison of our bending loss observations to theory is not possible because we know of no bending loss theory that applies to three-region guides with thin cladding, large refractive index difference between layers, and curvature radius as small as 20 guide wavelengths. In fact, all the theories with which we are familiar assume that the refractive index differences between layers are small. Keeping these limitations in mind, we have used the theory of Kuester and Chang [21], [22] for curved dielectric rods for rough comparison. To use the theory, the rod radius and dielectric constant were chosen equal to that of the core of the actual guide, and the surroundings of the rod were assumed to have a relative dielectric constant equal to one. The theoretical curvature losses were calculated for the vertically polarized $\mathrm{LP}_{10}$ mode, the "linearly polarized approximation" mode corresponding to the exact vertically polarized $\mathrm{HE}_{11}$ mode. Applied in this way, the theory predicted that a curvature radius of less than $2 \mathrm{~cm}$ would be necessary for our guides to exhibit bending losses comparable to our absorptive loses. We have obtained a similar result in comparing Marcatili's bending loss theory for rectangular dielectric guides [23] with experimental measurements of powder-filled channels in solid dielectric substrates [19], [24].

\section{CONCLUSIONS}

Guide wavelengths calculated using Kuhn's theory for a three-region lossless dielectric waveguide are in good agreement with experimental measurements at $10 \mathrm{GHz}$ and $94 \mathrm{GHz}$. A hybrid mode classification scheme for three-region guides based on the ratio $\left|E_{z} / H_{z}\right|$ seems more soundly based on physical properties and reduces to Snitzer's familiar scheme for two-region guides. Highly flexible guides consisting of low-loss, high-dielectric-constant powders packed inside thin-wall plastic tubing exhibit losses of the order of $10 \mathrm{~dB} /$ meter, low enough at 94 $\mathrm{GHz}$ to be attractive for short length transmission. Bending losses for $94 \mathrm{GHz}$ guide were negligible compared to absorptive losses for curvature radii greater than about 4 $\mathrm{cm}$.

\section{REFERENCES}

[1] D. Jablonski, "Attenuation characteristics of circular dielectric waveguide at millimeter wavelengths," IEEE Trans. Microwave Theory Tech., vol. MTT-26, pp. 667-671, Sept. 1978.

[2] Product brochure, W. R. Gore Co

[3] S. Shindo and I. Ohtomo, "A coaxial type dielectric waveguide for $100 \mathrm{GHz}$ band," Tech. Rep. IECE Japan, MW75-105, pp. 75-80, 1975.

[4] D. Gloge, "Weakly guiding fibers," Appl. Opt., vol. 10, pp. 2252-2258, Oct. 1971

[5] M. H. Kuhn, "The influence of the refractive index step due to the finite cladding of homogeneous fibers on the hybrid properties of modes," Arch. Elek. Übertragung. vol. 28, pp. 393-401, Oct. 1974.

[6] S. Ramo, J. R. Whinnery, and T. Van Duzer, Fields and Waves in Communication Electronics. New York: Wiley, 1966, ch. 7.

[7] H. G. Unger, Planar Optical Waveguides and Fibers. Oxford: Clarendon Press, 1977, ch. 4.

[8] A. Safaai-Jazi and G. L. Yip, "Classification of hybrid modes in cylindrical dielectric optical waveguides," Radio Sci., vol. 12, pp. 603-609, July-Aug. 1977.

[9] R. E. Beam, M. M. Astrahan, W. C. Jakes, H. M. Wachowski, and W. L. Firestone, "Dielectric tube waveguides," Rep. ATI 94929, ch. 5, Northwestern University, Evanston, IL, 1949.

[10] E. Snitzer, "Cylindrical dielectric waveguide modes," J. Opt. Soc. Amer., vol. 51, pp. 491-498, May 1961.

[11] N. S. Kapany and J. J. Burke, Optical Waveguides. New York: Academic Pres, 1972, pp. 118.

[12] P. J. B. Clarricoats, "Propagation along unbounded and bounded dielectric rods," Proc. Inst. Elec. Eng., vol. 108C, pp. 170-176, 1961.

[13] S. P. Schlesinger, P. Diament, and A. Vigants, "On higher-order hybrid modes of dielectric cylinders," Trans. Inst. Radio Eng., vol. MTT-8, no. 2, pp. 252, 1960.

[14] D. Marcuse, Light Transmission Optics. New York: Van Nostrand Reinhold, 1972, pp. 296-301.

[15] A. Safaai-Jazi and G. L. Yip, "Cutoff conditions in three-layer cylindrical dielectric waveguides," IEEE Trans. Microwave Theory Tech., vol. MTT-26, pp. 898-903, Nov. 1978.

[16] S. Roberts and A. R. von Hippel, "A new method for measuring dielectric constant and loss tangent in the range of centimeter waves," J. Appl. Phys., vol. 174 pp. 610-616, July 1946.

[17] A. R. von Hippel, Dielectric Materials and Applications. New York: Wiley, 1958, pp. 301-370.

[18] S. Ramo, J. R. Whinnery, and T. Van Duzer, Fields and Waves in Communication Electronics. New York: Wiley, 1966, pp. 432.

[19] W. M. Bruno, "Powder Core Dielectric Waveguides," Ph.D. thesis, California Institute of Technology, pp. 141-154, 1986. 
[20] W. B. Bridges, "Low loss flexible dielectric waveguide for millimeter-wave transmission and its application to devices," California Institute of Technology, Annual Tech. Rep. SRO-0005-1 on contract N00014-79-C-0839, ch. III, Mar. 1981.

[21] D. C. Chang and E. F. Kuester, "Radiation and propagation of a surface-wave mode on a curved open waveguide of arbitrary cross section," Radio Sci., vol. 11, pp. 449-457, May 1976.

[22] E. F. Kuester and D. C. Chang, "Surface-wave radiation loss from curved dielectric slabs and fibers," IEEE J. Quantum Electron., vol QE-11, pp. 903-907, Nov. 1975.

[23] E. A. J. Marcatili, "Bends in optical dielectric guides," Bell Syst. Tech. J., vol. 48, pp. 2103-2132, Sept. 1969.

[24] W. M. Bruno and W. B. Bridges, "Properties of millimeter-wave dielectric waveguide made with powder-filled channels in a dielectric substrate," in preparation.

事

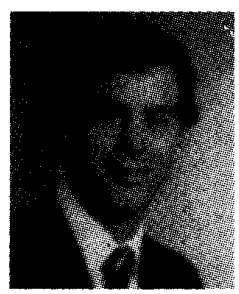

William M. Bruno (S'82-M'86) was born in Ithaca, NY, in 1958. He received the B.S. in electrical engineering from the University of California at San Diego in 1981 and the M.S. and $\mathrm{Ph} . \mathrm{D}$. degrees in electrical engineering from the California Institute of Technology in 1983 and 1986 , respectively.

His graduate research at Caltech focused on millimeter-wave dielectric waveguides. Since joining TRW in 1985 as a Member of the Technical Staff, he has been working in the area of

optical communications.

Dr. Bruno is a member of Phi Beta Kappa.

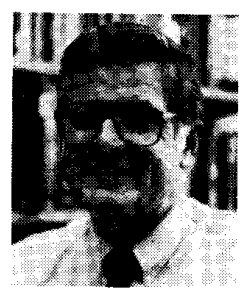

William B. Bridges (S'53-M'61-F'70) was born in Inglewood, CA, in 1934 . He received the B.S.

M.S., and Ph.D. degrees in electrical engineering from the University of California, Berkeley, in 1956,1957 , and 1962, respectively, and was an Associate in Electrical Engineering from 1957 to 1959 , teaching courses in communication and circuits. His graduate research dealt with noise in microwave tubes and electron-stream instabilities. Summer jobs at RCA and Varian provided stimulating experience with microwave radar systems, ammonia beam masers, and the early development of the ion vacuum pump.

He joined the Hughes Research Laboratories in 1960 as a member of the Technical Staff and was a Senior Scientist from 1968 to 1977, with a brief tour as Manager of the Laser Department in 1969-70. His research at Hughes involved gas lasers of all types and their application to optical communication, radar, and imaging systems. He is the discoverer of laser oscillation in noble gas ions and spent several years on the engineering development of practical high-power visible and ultraviolet ion lasers for military applications. He joined the faculty of the California Institute of Technology in 1977 as Professor of Electrical Engineering and Applied Physics. He served as Executive Officer for Electrical Engineering from 1979 to 1981. In 1983, he was appointed Carl F. Braun Professor of Engineering and currently conducts research in gas lasers and millimeter/submillimeter-wave technology.

Dr. Bridges is member of Eta Kappa Nu, Tau Beta Pi, Phi Beta Kappa and Sigma $\mathrm{Xi}$, receiving Honorable Mention from Eta Kappa $\mathrm{Nu}$ as "Outstanding Young Electrical Engineer" in 1966, the Distinguished Teaching award in 1980 and 1982 from the Associated Students of Caltech, and the Arthur L. Schawlow Medal from the Laser Institute of America in 1986. He is a member of the National Academy of Engineering and the National Academy of Sciences and a Fellow of the Optical Society of America. He was a Sherman Fairchild Distinguished Scholar at Caltech in 1974-75. He is coauthor (with C. K. Birdsall) of Electron Dynamics of Diode Regions (New York: Academic Press, 1966.) He has served on various committees of the IEEE and OSA, and was formerly Associate Editor of the IEEE JOURNAL OF QUANTUM ELECTRONICS and the Journal of the Optical Society of America. He currently serves as President of the Optical Society of America. 